\title{
SOME REMARKS ON ONE-SIDED INVERSES
}

N. JACOBSON

Let $\mathfrak{A}$ be an arbitrary ring with an identity 1 , and suppose that $\mathfrak{A}$ contains a pair of elements $u, v$ such that

$$
u v=1 \text { but } v u \neq 1 \text {. }
$$

We introduce the elements

$$
e_{i, j}=v^{i-1} u^{j-1}-v^{i} u^{j}
$$

for $i, j=1,2,3, \cdots$, where it is understood that $u^{0}=1=v^{0}$. It can be verified directly that the $e_{i j}$ thus defined satisfy the multiplication table for matrix units:

$$
e_{i j} e_{r s}=\delta_{j r} e_{i s} .
$$

In particular the elements $e_{i}=e_{i i}$ are orthogonal idempotent elements. No $e_{i j}=0$. For by (3) the vanishing of one of the $e_{i j}$ implies the vanishing of all; in particular, it implies that

$$
0=e_{1}=1-v u
$$

contrary to (1).

The existence of an infinite set of orthogonal idempotent elements in a ring $\mathscr{A}$ is incompatible with mild chain conditions on the ring. If $\left\{e_{i}\right\}$ is such a set of idempotent elements and we set $f_{k}=\sum_{1}^{k} e_{i}$, then

$$
f_{1} \mathfrak{A} \subset f_{2} \mathfrak{A} \subset f_{3} \mathfrak{A} \subset \cdots
$$

is an infinite properly ascending chain of right ideals. The right annihilator of an idempotent element $f$ is the set of elements $\{a-f a\}$. If $\mathfrak{A}$ has an identity, this right ideal is the principal right ideal $(1-f) \mathfrak{A}$. Even if $\mathfrak{A}$ does not have an identity, it is customary to denote the set $\{a-f a\}$ as $(1-f)$ भ. It is clear that the following is an infinite properly descending chain of annihilators

$$
\left(1-f_{1}\right) \mathfrak{A} \supset\left(1-f_{2}\right) \mathfrak{A} \supset\left(1-f_{3}\right) \mathfrak{A} \supset \cdots \text {. }
$$

Our remarks imply the following theorem which includes a result due to Baer. ${ }^{1}$

THEOREM 1. If $\mathfrak{A}$ is a ring with an identity that satisfies either the

Received by the editors April 14, 1949.

1 R. Baer, Inverses and zero-divisors, Bull. Amer. Math. Soc. vol. 48 (1942) pp. 630-638. 
ascending or the descending chain condition for principal right ideals generated by idempotent elements, then $u v=1$ in $\mathfrak{A}$ implies $v u=1$.

If $e_{11}, e_{12}, e_{21}, e_{22}$ are elements of a ring satisfying (3), then $e_{11} \mathfrak{A}=e_{12} \mathfrak{A}$ and the mapping $x \rightarrow e_{12} x$ is an $\mathfrak{Y}$-isomorphism of $e_{22} \mathfrak{A}$ onto $e_{11} \mathfrak{A}$. Hence if the $e_{i i}$ are defined as above, then the right ideals $e_{i i} \mathfrak{A}$ are $\mathfrak{A}$-isomorphic. The right ideal $\mathfrak{B}=\sum e_{i i} \mathfrak{A}$ is a direct sum of the $e_{i i} \mathfrak{A}$. Hence we have the following result.

THEOREM 2. If $\mathfrak{A}$ is a ring with an identity that contains two elements $u$ and $v$ such that $u v=1, v u \neq 1$, then $\mathfrak{A}$ contains a right ideal that is a direct sum of an infinite number of $\mathfrak{A}-i$ somorphic right ideals.

We note next a result that was proved first by Kaplansky (oral communication) using structure theory.

THEOREM 3. If an element of a ring with an identity has more than one right inverse, then it has an infinite number of right inverses.

PROOF. If $v$ is one of the right inverses of the element $u$, then we have $u v=1, v u \neq 1$. If the $e_{i j}$ are defined as above, then $u e_{11}=u(1-v u)$ $=0$. Hence also $u e_{1 k}=0$ for $k=1,2,3, \cdots$. If $e_{1 k}=e_{1 l}$ for $k \neq l$, then $e_{1 k} e_{k k}=e_{12} e_{k k}$ and $e_{1 k}=0$. Hence the $e_{1 k}$ are all different and the elements $v_{k}=v+e_{1 k}$ are all different. Evidently $u v_{k}=1$.

We assume next that $\mathfrak{A}$ is an algebra over a field $\Phi$ with an identity and that $\mathfrak{A}$ contains elements $u, v$ satisfying (1). We wish to determine the structure of the algebra $\Phi[u, v]$ generated by $u$ and $v$. For this purpose we introduce a vector space $\Re$ that has a denumerable basis $\left(x_{1}, x_{2}, x_{3}, \cdots\right)$ over $\Phi$. Let $\bar{U}$ and $\bar{V}$, respectively, be the linear transformations in $\Re$ that have the matrices

$$
U=\left(\begin{array}{ccccc}
0 & 1 & & & \\
0 & 0 & 1 & & \\
0 & 0 & 0 & 1 & \\
& & & & \ddots
\end{array}\right), \quad V=\left(\begin{array}{cccc}
0 & 0 & 0 & \ldots \\
1 & 0 & 0 & \ldots \\
0 & 1 & 0 & \ldots \\
& & 1 & \\
& & &
\end{array}\right)
$$

relative to the given basis. We have the relations $U V=1, V U \neq 1$. Hence any element in $\Phi[U, V]$ is a linear combination of the elements $V^{i} U^{i}, i, j,=0,1,2, \cdots$. We shall now show that these elements are linearly independent; hence they form a basis. We can verify that

$$
V^{i} U^{i}=\operatorname{diag}\{0,0, \cdots, 0 ; 1,1, \cdots\}
$$


where there are $i$ zeros. Hence $V^{i} U^{i+k}$ has nonzero elements only in the $k$ th super-diagonal and $V^{i+k} U^{i}$ has nonzero elements only in the $k$ th sub-diagonal. Any relation $\sum \beta_{i j} V^{i} U^{i}=0$ therefore implies that

$$
\sum_{i} \beta_{i, i+k} V^{i} U^{i+k}=0, \quad \sum_{i} \beta_{i+k, i} V^{i+k} U^{i}=0 .
$$

Multiplication of the first of these equations on the right by $V^{k}$ gives

$$
\sum \beta_{i, i+k} V^{i} U^{i}=0 .
$$

It is evident from (7) that the matrices $1=V^{0} U^{0}, V^{1} U^{1}, \ldots$ are linearly independent. Hence $\beta_{i, i+k}=0$. Similarly every $\beta_{i+k, k}=0$. This proves our assertion.

The matrices

$$
E_{i, j}=\left(V^{i-1} U^{j-1}-V^{i} U^{i}\right)
$$

are the usual matrix units. Hence $\Phi[U, V]$ contains every matrix of the form

$$
\left(\begin{array}{ll}
A & 0 \\
0 & 0
\end{array}\right)
$$

where $A$ is a finite square matrix. Using (8), we can express any $V^{i} U^{i}$ in the form $\sum \beta_{r s} E_{r s}+\phi(U)+\psi(V), \phi$ and $\psi$ polynomials.

Now it is clear that the subalgebra of $\Phi[\bar{U}, \bar{V}]$ corresponding to the algebra of matrices (9) is a dense algebra of linear transformations of finite rank. ${ }^{2}$ Also it is easy to see that $\phi(\bar{U})+\psi(\bar{V})$ has infinite rank unless $\phi$ and $\psi$ are 0 . Hence the transformations with matrices (9) constitute the complete set of linear transformations of finite rank in $\Phi[U, V]$. It follows from known structure results that $\Phi[\bar{U}, \bar{V}]$ (and $\Phi[U, V])$ is a primitive algebra that has minimal one-sided ideals. ${ }^{3}$ Moreover, the subalgebra corresponding to (9) is the minimal twosided ideal of this algebra. Any nonzero two-sided ideal contains this one, and in particular it contains the elements $1-\bar{V} \bar{U}$.

Since the linear transformations $\bar{V}^{i} \bar{U}^{i}$ are linearly independent it is clear that the mapping $\bar{U} \rightarrow u, \bar{V} \rightarrow v$ can be extended to a homomorphism of $\Phi[\bar{U}, \bar{V}]$ onto $\Phi[u, v]$. Since $v u \neq 1$, the kernel of this homomorphism does not include $1-\bar{V} \bar{U}$. Hence it is 0 and our correspondence is an isomorphism. This completes the proof of the following theorem:

Theorem 4. Any two algebras $\Phi\left[u_{i}, v_{i}\right], i=1,2$, in which

2 Cf. the author's, The radical and semi-simplicity for arbitrary rings, Amer. J. Math. vol. 47 (1945) p. 313.

${ }^{3}$ See the reference cited in footnote 2, p. 317 . 
$u_{i} v_{i}=1, v_{i} u_{i} \neq 1$ are isomorphic under an isomorphism that pairs the $u_{i}$ and the $v_{i}$. The algebras $\Phi\left[u_{i}, v_{i}\right]$ are primitive algebras that have minimal one-sided ideals.

The minimal two-sided ideal $\mathfrak{B}$ of $\Phi[u, v]$ is the infinite matrix algebra with basis $e_{i j}$. Any element of $\Phi[u, v]$ is congruent $\bmod \mathfrak{B}$ to an element of the form $\phi(u)+\psi(v)$. It follows that $\Phi[u, v] / \mathscr{B}$ is isomorphic to the group algebra of an infinite cyclic group.

Suppose now that $\Re$ is any vector space over $\Phi$ and that $\bar{U}$ and $\bar{V}$ are linear transformations in $\Re$ over $\Phi$ such that $\bar{U} \bar{V}=1, \bar{V} \bar{U} \neq 1$. Let $\bar{B}$ denote the minimal two-sided ideal in $\Phi[\bar{U}, \bar{V}]$. Then $\bar{B}$ has the basis $\bar{E}_{i j}$ defined as in (2). It follows easily that $\bar{B}$ is a direct sum of the right ideals $\bar{\Im}_{k}=\bar{E}_{k k} \Phi[\bar{U}, \bar{V}]$ and that the $\bar{\Im}_{k}$ are minimal: If $x \in \Re$, the subspace $x \bar{\Im}_{k}$ is either 0 or it is $\Phi[\bar{U}, \bar{V}]$-isomorphic to $\bar{\Im}_{k}$. In the latter case $x \bar{\Im}_{k}$ is irreducible. It follows that the subspace $\Re \bar{B}$ can be decomposed as a direct sum of subspaces that are invariant and irreducible relative to $\bar{U}$ and $\bar{V}$. It is easy to see that all of these spaces are isomorphic and that if suitable bases are chosen in these spaces, then the matrices $U$ and $V$ have the form (6). The factor space $\mathfrak{S}=\Re-\Re \overline{\mathfrak{B}}$ is annihilated by $\overline{\mathfrak{B}}$. Hence the induced transformations $\bar{U}$ and $\bar{V}$ in this space satisfy $\bar{U} \bar{V}=1=\bar{V} \bar{U}$.

Nearly all of our results hold also for quasi-inverses. In any ring $\mathfrak{A}$ we define $a \circ b=a+b-a b$. Then $\mathfrak{A}$ is a semigroup relative to this composition and 0 is the identity. An element $b$ is a right quasiinverse of $a$ if $a \circ b=0$. If $\mathfrak{A}$ has an identity $1,(1-a)(1-b)=1$ $-a \circ b$, so that if $a \circ b=0$, then $(1-a)(1-b)=1$ and conversely. Now suppose that $\mathfrak{A}$ contains two elements $a$ and $b$ such that

$$
a \circ b=0, \quad b \circ a \neq 0 .
$$

If we define $x^{\circ k}=x^{\circ k-1} \circ x$, then we can verify that the elements

$$
e_{i, j}=b^{\circ i} \circ a^{\circ j}-b^{\circ i-1} \circ a^{\circ j-1}
$$

satisfy the multiplication for matrix units. All of the $e_{i j}$ are nonzero. In particular, $\mathfrak{A}$ contains an infinite number of orthogonal idempotent elements. Then we see that if $\mathfrak{A}$ satisfies the ascending chain condition on principal ideals generated by idempotent elements, then $a \circ b=0$ in $\mathscr{A}$ implies $b \circ a=0 .{ }^{4}$ This is the analogue of Theorem 1. Theorems 2,3 , and 4 carry over without change.

\section{YALE UNIVERSITY}

4 Baer's results cited in footnote 1 have been extended to quasi-inverses by Andrunakievic in his paper Semi-radical rings, Izvestiya Akademii Nauk SSSR. Ser. Mat. vol. 12 (1948) pp. 129-178. 